\title{
Mega United States Pharmacopeia Unit
}

National Cancer Institute

\section{Source}

National Cancer Institute. Mega United States Pharmacopeia Unit. NCI Thesaurus. Code C73571.

A unit of potency equal to one million US Pharmacopoeia Units. 